Research Paper

\title{
Albumin-to-Alkaline Phosphatase Ratio as an Independent Prognostic Factor for Overall Survival of Advanced Hepatocellular Carcinoma Patients without Receiving Standard Anti-Cancer Therapies
}

\author{
Xiurong Cai ${ }^{1 *}$, Zhanhong Chen ${ }^{1,2 * \square}$, Jie Chen ${ }^{1 *}$, Xiaokun $\mathrm{Ma}^{1}$, Mingjun Bai ${ }^{3}$, Tiantian Wang ${ }^{1}$, Xiangwei

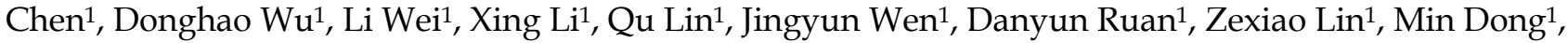 \\ Xiangyuan $\mathrm{Wu}^{1 \mathrm{~W}}$ \\ 1. Department of Medical Oncology and Guangdong Key Laboratory of Liver Disease, the Third Affiliated Hospital of Sun Yat-sen University, 600 Tianhe \\ Road, Guangzhou, 510630, People's Republic of China; \\ 2. Department of Medical Oncology of Sun Yat-sen University Cancer Center, State Key Laboratory of Oncology in South China, Collaborative Innovation \\ Center for Cancer Medicine, 651 Dongfengdong Road, Guangzhou, 510060, People's Republic of China; \\ 3. Department of Intervention and Radiology, Guangdong Key Laboratory of Liver Disease, the Third Affiliated Hospital of Sun Yat-sen University, 600 \\ Tianhe Road, Guangzhou, 510630, People's Republic of China. \\ * These authors contributed equally to this work
}

$\square$ Corresponding authors: Zhanhong Chen: Address: Department of Medical Oncology and Guangdong Key Laboratory of Liver Disease, the Third Affiliated Hospital of Sun Yat-sen University, 600 Tianhe Road, Guangzhou, 510630, People's Republic of China; Tel: +86-20-85252212; E-mail: chzhanh3@mail.sysu.edu.cn Xiangyuan Wu: Address: Department of Medical Oncology and Guangdong Key Laboratory of Liver Disease, the Third Affiliated Hospital of Sun Yat-sen University, 600 Tianhe Road, Guangzhou, 510630, People’s Republic of China; Tel: +86-20-85252212; E-mail: wuxiangy@mail.sysu.edu.cn

(c) Ivyspring International Publisher. This is an open access article distributed under the terms of the Creative Commons Attribution (CC BY-NC) license (https://creativecommons.org/licenses/by-nc/4.0/). See http://ivyspring.com/terms for full terms and conditions.

Received: 2017.07.06; Accepted: 2017.10.24; Published: 2018.01.01

\begin{abstract}
Background Albumin-to-Alkaline Phosphatase Ratio (ALB/ALP ratio, AAPR), a newly developed index of liver function, has been rarely discussed about its prognostic value in malignancies. The current study attempted to evaluate the prognostic prediction of AAPR in advanced HCC.

Methods 237 advanced HCC patients who refused any standard anti-cancer therapies were retrospectively analyzed. The threshold value of AAPR was determined by receiver operating characteristic (ROC) curve. Univariate analyses using Kaplan-Meier method and log-rank test, and multivariate analysis using Cox proportional hazards regression model were conducted. Comparisons of ROC curves and likelihood ratio test (LRT) were utilized to compare the value of different factors in predicting survival.

Results ROC curve analysis confirmed 0.38 as the optimal cutoff value of AAPR in evaluating overall survival (OS). Patients with an AAPR $>0.38$ exhibited significantly lower frequencies of ascites, portal vein tumor thrombus, Child-Pugh grade B \& C, and KPS $<70$ (all $P<0.05$ ). These patients also displayed a longer median survival time than those with an AAPR $\leq 0.38(5.8 \mathrm{~m}$ vs $2.4 \mathrm{~m}, P<0.01)$. Univariate and multivariate analyses identified AAPR as an independent prognostic indicator $(H R=0.592, P=0.007)$. Furthermore, we integrated AAPR with TNM system and found that area under curve of AAPR-TNM system was significantly larger than that of TNM system when predicting 3-month survival $(0.670$ vs $0.611, P<0.01)$. Moreover, LRT indicated that AAPR-TNM system had a significantly larger $\chi^{2}(26.4 \mathrm{vs}$ $16.4, P<0.01)$ and a significantly smaller Akaike information criterion value $(1936$ vs $1948, P<0.01)$ comparing with TNM system.

Conclusions Our study implied that AAPR was a potentially valuable prognostic index for advanced HCC patients without receiving any standard anti-cancer therapies. AAPR-TNM system preceded TNM system in predicting overall survival in this study.
\end{abstract}

Key words: advanced hepatocellular carcinoma; albumin-to-alkaline phosphatase ratio; prognosis; hepatic function reserve; biomarkers. 


\section{Introduction}

Hepatocellular carcinoma (HCC) is the second primary cause of malignancy-related mortality in males and the sixth in females, giving rise to approximately 745,500 deaths worldwide [1]. Hepatic resection and liver transplantation are effective curative treatment modalities for HCC in conditions of localized tumors originating from non-cirrhotic liver or from cirrhotic liver with adequate liver function [2]. Due to limited specific symptoms, most HCC patients in China are initially diagnosed in advanced stage, which cannot be treated by surgery [3]. Though with robust development of local interventional treatments, targeted agents (sorafenib) and immunotherapies, the outcomes of these treatments remain heterogeneous in advanced HCC [4-8]. Therefore, prognostic factors or biomarkers which help survival prediction and clinical decision-making, are still urgent to be studied nowadays. Clinicopathological features including extrahepatic tumor lesions, portal vein tumor thrombus (PVTT), microvascular invasion and tumor-infiltrating lymphocytes were reported as prognostic factors for HCC [9, 10]. Furthermore, multifarious serum and tissue biomarkers have been investigated for their potential prognostic value in HCC, which includes alpha-fetoprotein (AFP), albumin (ALB), bilirubin, alkaline phosphatase (ALP), vascular endothelial growth factor (VEGF) and soluble VEGF receptor-1 (sVEGFR-1) [11-14].

In addition to prognostic factors or biomarkers, staging systems have prognostic values as well. More than ten staging systems have been defined, and some of them have been applied to predicting survival and guiding therapeutic regimen for patients with HCC. The most widely applied staging systems include the Barcelona Clinic Liver Cancer (BCLC) system, and the American Joint Committee on Cancer (AJCC) TNM staging system [15-17].

To our consensus, the outcomes of patients with HCC relies on tumor burden, residual hepatic function, performance status and treatment modalities. As an index related to liver function, albumin-to-alkaline phosphatase ratio (AAPR) is a newly noticeable marker in predicting survival of malignancies [18]. Only one study reported AAPR as an independent prognostic indicator for HCC patients receiving surgery and palliative therapy [19]. Nevertheless, its prognostic value in advanced HCC patients without undergoing any standard anti-cancer therapy, remains unknown. This cohort of patients exhibits a natural progress of HCC, which deserves our careful study for more valuable factors predicting survival.
In present study, we for the first time analyzed a group of advanced HCC cases without undergoing any standard anti-cancer treatments, and investigated the prognostic significance of AAPR and its association with other clinicopathological features.

\section{Methods}

\section{Patient selection and evaluation}

We retrieved medical records of the patients who were diagnosed with HCC and hospitalized in The Third Affiliated Hospital of Sun Yat-sen University from June 2006 to October 2010.

Inclusion criteria were as follows: (1) diagnosis as advanced HCC was confirmed by pathological examination or conformed to radiological criteria from the American Association for the Study of Liver Diseases [20], and exhibited stage C or D according to BCLC system; (2) without receiving any standard anti-cancer therapies recommended by the National Comprehensive Cancer Network (NCCN) guidelines, according to their own wills and decisions [21]. Exclusion criteria were as follows: (1) incomplete baseline metabolic panel data; (2) synchronal malignancies; (3) being lost to follow-up within six months.

According to the NCCN guidelines, patients were regularly followed up every 3-6 months for 2 years, then every 6-12 months in either outpatient clinic or inpatient department. Additionally, we conducted telephone interviews to those who did not come back to our hospital.

Written informed consent was obtained from the included patients or their family members. The clinical ethics committee of The Third Affiliated Hospital of Sun Yat-sen University (Guangzhou, China) approved all procedures conducted in present study, which conformed to the norm of the Declaration of Helsinki.

\section{Data collection}

Patients' clinical information was reviewed and acquired from the hospital database. The overall survival (OS) time, defined as the time from diagnosis to death of any cause or to the date of the last follow-up, was set as our primary outcome.

Medical history, physical and laboratory examination, and imaging tests were collected at the time of their initial diagnosis of advanced HCC, then were analyzed for baseline evaluation. Tumor-related characteristics including the number of tumor nodules, maximum tumor diameter, PVTT and distant metastasis were acquired from medical imaging tests. At the time of initial diagnosis of advanced HCC, serum levels of ALB and ALP were derived from hepatic biochemistry examination, 
which was measured following the recommended procedures of the automatic biochemical analyzer. The AAPR was computed by dividing the serum ALB level by the serum ALP level, which ALB is in $\mathrm{g} / \mathrm{L}$ and ALP in U/L.

\section{Statistical analysis}

Chi-square test and Fisher's exact test (two-tailed) were applied to compare difference of categorical variables between groups, and Mann-Whitney test was used for those between medians. By setting OS as the endpoint, receiver operating characteristic (ROC) curve was utilized to determine the optimal threshold value of AAPR as the Youden index reached its maximum [22]. Consequently, patients were distributed into either a low-AAPR group or a high-AAPR group. Kaplan-Meier method and log-rank test were conducted in univariate analyses for estimation of survival data between/among different groups. Subsequently, those significant factors were then analyzed by a Cox proportional hazards regression model (backward method) to distinguish potentially independent prognostic factors. Comparisons of ROC curves and likelihood ratio test (LRT) were conducted to compare the ability of different factors in predicting OS. All statistical analyses were accomplished by using SPSS (version 24.0; IBM Corp., Armonk, NY, USA), Medcalc (version 15.8; MedCalc Software bvba, Acacialaan, Belgium) and Graphpad Prism 5 (GraphPad Software Inc., La Jolla, CA, USA). A $P$ value less than 0.05 was taken for indication of statistical significance.

\section{Results}

\section{Patient characteristics}

As shown in Table 1, a total of 237 patients were included, with a median age of 56 years [Interquartile range (IQR) 45-66 years]. The number of male patients far exceeded that of female ones [206 vs $31(86.9 \%$ vs $13.1 \%)] .129$ (54.4\%) and six (2.5\%) HCC patients were diagnosed with ascites and hepatic encephalopathy, respectively. The median Karnofsky performance status (KPS) was 70 (IQR 20-80). As for laboratory parameters, the median levels of AFP, blood urea nitrogen (BUN), ALB and ALP were $629.0 \mathrm{ng} / \mathrm{dL}, 5.1$ $\mathrm{mmol} / \mathrm{L}, 34.5 \mathrm{~g} / \mathrm{L}$ and $149.5 \mathrm{U} / \mathrm{L}$, respectively. The median AAPR of all patients was 0.23 (IQR 0.14-0.33). $112(47.2 \%)$ patients exhibited Child-Pugh grade B. As for the tumor characteristics, $180(75.9 \%)$ patients with tumor diameter larger than $5 \mathrm{~cm}, 49$ (20.7\%) with distant metastasis and $158(66.7 \%)$ with PVTT were recorded at the time of diagnosis. The 3-month, 6-month and 12-month survival rates of patients in present study were $49.4 \%, 31.2 \%$ and $17.7 \%$, respectively.

Table 1. Baseline demographics of 237 patients with advanced $\mathrm{HCC}$ in present study.

\begin{tabular}{ll}
\hline Characteristic & Total \\
\hline Age, years (median/IQR) & $56(45-66)$ \\
Gender, $\mathrm{n}(\%)$ & \\
Male & $206(86.9)$ \\
Female & $31(13.1)$ \\
Ascites, $\mathrm{n}(\%)$ & $129(54.4)$ \\
Hepatic encephalopathy, $\mathrm{n}(\%)$ & $6(2.5)$ \\
KPS (median/IQR) & $70(20-80)$ \\
Laboratory parameters (median/IQR) & \\
AFP, ng/dL & $629.00(22.20-1180.00)$ \\
BUN, mmol/L & $5.10(3.95-7.07)$ \\
ALB, g/L & $34.50(29.50-38.45)$ \\
ALP, U/L & $149.50(100.00-228.00)$ \\
AAPR & $0.23(0.14-0.33)$ \\
Child-Pugh grade, $\mathrm{n}(\%)$ & \\
A & $67(28.3)$ \\
B & $112(47.2)$ \\
C & $58(24.5)$ \\
TNM (AJCC 7th), $\mathrm{n}(\%)$ & \\
I & $28(11.8)$ \\
II & $21(8.9)$ \\
III & $136(57.4)$ \\
IV & $52(21.9)$ \\
Distant metastasis, $\mathrm{n}(\%)$ & $49(20.7)$ \\
Portal vein tumor thrombus, $\mathrm{n}(\%)$ & $158(66.7)$ \\
Tumor size (cm), $\mathrm{n}(\%)$ & \\
55 & $57(24.1)$ \\
$>5$ & $180(75.9)$ \\
survival rate, $\mathrm{n}(\%)$ & \\
3-month & $117(49.4)$ \\
6-month & $74(31.2)$ \\
12-month & $42(17.7)$ \\
\hline &
\end{tabular}

IQR: interquartile range; KPS: Karnofsky performance status; AFP: alpha fetoprotein; BUN: blood urea nitrogen; ALB: albumin; ALP: alkaline phosphatase; AAPR: albumin-to-alkaline phosphatase ratio; TNM: Tumor-node-metastasis; AJCC: American Joint Committee on Cancer.

Interestingly, different levels of AAPR were found in a series of subsets when we stratified patients according to their different status of ascites, PVTT, Child-Pugh grade and KPS. As shown in Figure 1, the AAPR was significantly lower in patients with ascites than those without $(0.186$ vs $0.291, P<$ $0.001)$, with PVTT than those without (0.201 vs 0.287 , $P<0.001$ ), with Child-Pugh grade $B$ and $C$ than those with grade A ( 0.189 vs $0.338, P<0.001)$, and with KPS $<70$ than those with KPS $\geq 70(0.208$ vs $0.238, P=$ 0.009 ).

\section{Comparison of patients between the low-AAPR and high-AAPR groups}

ROC curve was performed with the OS setting as the state variable and with AAPR setting as the test variable for OS. Consequently, the optimal threshold value for AAPR was 0.38 with $85.5 \%$ sensitivity and $39.1 \%$ specificity. Patients were dichotomized into 
either the low-AAPR group (AAPR $\leq 0.38$ ) or the high-AAPR group (AAPR > 0.38) afterwards.

Finally, 195 and 42 patients were included in the low- and the high-AAPR groups, respectively. Clinicopathological characteristics between the groups were balanced for the most parts except the

A

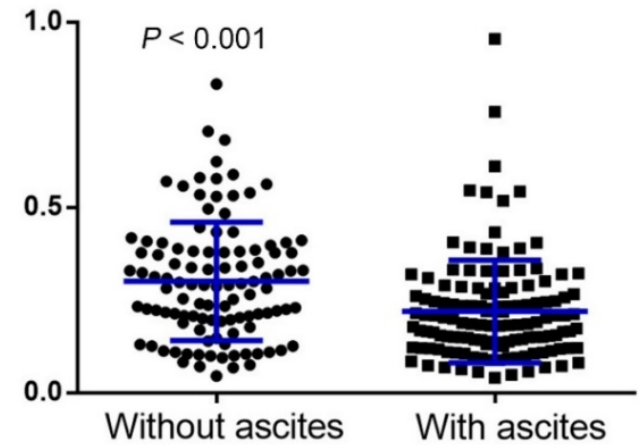

C

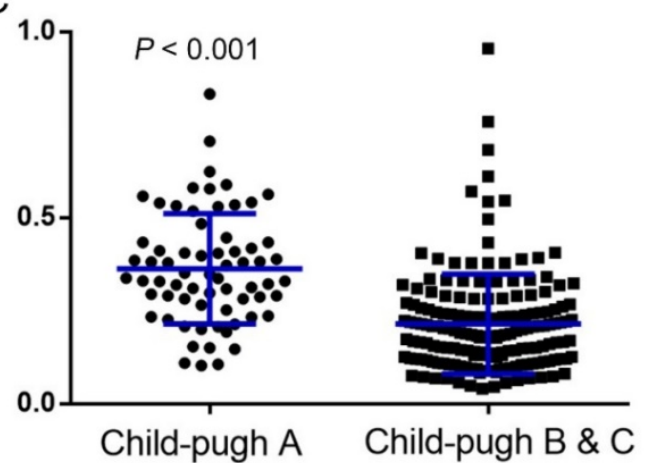

status of ascites, PVTT, Child-Pugh grade and KPS (Table 2). Consistent with our previous results, patients in low-AAPR group exhibited significantly higher frequencies of ascites, PVTT, Child-Pugh grade $B$ and $C$, and KPS $<70$ (all $P<0.05$ ).

B

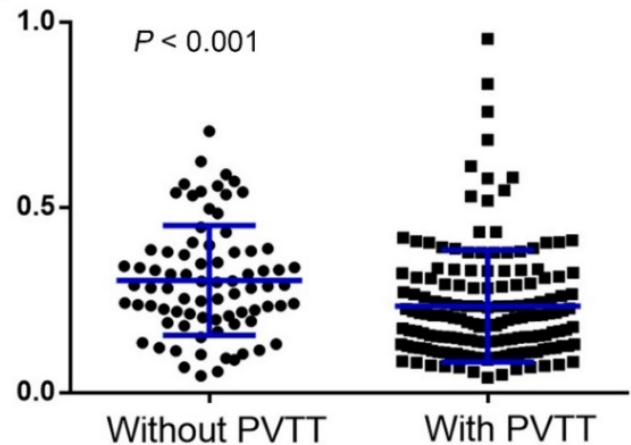

D

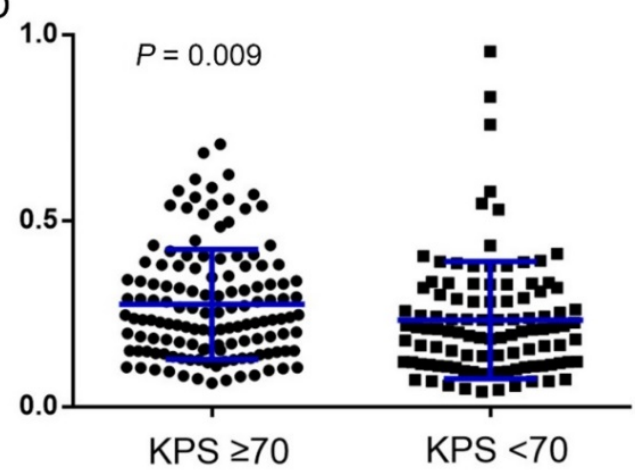

Figure 1. Pretreatment AAPR levels were compared using Mann-Whitney test between subgroups dichotomized by: (A) ascites (without vs with); (B) PVTT (without vs with); (C) Child-Pugh grade (grade A vs grade B\&C); and (D) KPS $(\geq 70$ vs $<70$ ), respectively (all $P<0.05$ ). The median and the corresponding interquartile range of each group were signified by the blue lines within the scatter plots. PVTT: portal vein tumor thrombus; KPS: Karnofsky performance status.

Table 2. Chi-square analysis for clinicopathologic variables in low- and high-AAPR groups.

\begin{tabular}{|c|c|c|c|}
\hline Variable & $\begin{array}{l}\mathrm{AAPR} \leq 0.38 \\
\mathrm{n}(\%)\end{array}$ & $\begin{array}{l}\text { AAPR }>0.38 \\
n(\%)\end{array}$ & $P$ value \\
\hline Age, years $(>50 / \leq 50)$ & $124 / 71(63.6 / 36.4)$ & $29 / 13(69.0 / 31.0)$ & 0.502 \\
\hline Gender (male/female) & $168 / 27(86.2 / 13.8)$ & $38 / 4(90.5 / 9.5)$ & 0.451 \\
\hline Ascites (yes/no) & $116 / 79(59.5 / 40.5)$ & $13 / 29(31.0 / 69.0)$ & 0.001 \\
\hline Encephalopathy (yes/no) & $6 / 189(3.1 / 96.9)$ & $0 / 42(0 / 100.0)$ & 0.250 \\
\hline $\operatorname{KPS}(\geq 70 /<70)$ & $58 / 137(29.7 / 70.3)$ & $23 / 19(54.8 / 45.2)$ & 0.002 \\
\hline \multicolumn{4}{|l|}{ Laboratory parameters } \\
\hline $\mathrm{AFP}, \mathrm{ng} / \mathrm{dL}(>400 / \leq 400)$ & $103 / 92(52.8 / 47.2)$ & $22 / 20(52.4 / 47.6)$ & 0.959 \\
\hline BUN, mmol/L (>8.9/ $\leq 8.9)$ & $27 / 168(13.8 / 86.2)$ & $3 / 39(7.1 / 92.9)$ & 0.236 \\
\hline ALB, $g / L(>36 / \leq 36)$ & $56 / 139(28.7 / 71.3)$ & $31 / 11(73.8 / 26.2)$ & $<0.001$ \\
\hline ALP, U/L (>200/ $\leq 200)$ & $70 / 125(35.9 / 64.1)$ & $0 / 42(0 / 100.0)$ & $<0.001$ \\
\hline Child-Pugh grade (A/B/C) & $39 / 102 / 54(20.0 / 52.3 / 27.7)$ & $28 / 10 / 4(66.7 / 23.8 / 9.5)$ & $<0.001$ \\
\hline TNM stage (III-IV/I-II) & $157 / 38(80.5 / 19.5)$ & $31 / 11(73.8 / 26.2)$ & 0.331 \\
\hline Distant metastasis (yes/no) & $40 / 152(20.8 / 79.2)$ & $9 / 32(22.0 / 78.0)$ & 0.873 \\
\hline PVTT (yes/no) & $136 / 59(69.7 / 30.3)$ & $22 / 20(52.4 / 47.6)$ & 0.030 \\
\hline Tumor size $(>5 \mathrm{~cm} / \leq 5 \mathrm{~cm})$ & $151 / 44(77.4 / 22.6)$ & $29 / 13(69.0 / 31.0)$ & 0.249 \\
\hline 3-months (survival/death) & $83 / 112(42.6 / 57.4)$ & $34 / 8(81.0 / 19.0)$ & $<0.001$ \\
\hline 6-months (survival/death) & $55 / 140(28.2 / 71.8)$ & $19 / 23(45.2 / 44.8)$ & 0.031 \\
\hline 12-months (survival/death) & $30 / 165(15.4 / 84.6)$ & $12 / 30(28.6 / 71.4)$ & 0.042 \\
\hline
\end{tabular}

KPS: Karnofsky performance status; AFP: alpha fetoprotein; BUN: blood urea nitrogen; ALB: albumin; ALP: alkaline phosphatase; AAPR: albumin-to-alkaline phosphatase ratio; TNM: tumor-node-metastasis; AJCC: American Joint Committee on Cancer; PVTT: portal vein tumor thrombus. 


\section{Survival analysis}

The 3-month, 6-month and 12-month survival rates were $42.6 \%, 28.2 \%$ and $15.4 \%$ in low-AAPR group, while $81.0 \%, 45.2 \%$ and $28.6 \%$ in high-AAPR group, respectively. Patients in low-AAPR group displayed a shorter median OS time than those in high-AAPR group $(2.4 \mathrm{~m}$ vs $5.8 \mathrm{~m}, P<0.01)$. To explore the prognostic factors for OS, the survival curves were stratified by gender, age, ascites, KPS, AFP, BUN, ALB, ALP, Child-Pugh grade, T category, lymph node metastasis, distant metastasis, PVTT and AAPR, respectively (Figure 2). As shown in the univariate analyses, all factors above were significant indicators for OS (all $P<0.05)$ except gender $(P=$ $0.111), \operatorname{KPS}(P=0.175), \operatorname{ALB}(P=0.549)$, and distant metastasis $(P=0.195)$ (Table 3$)$.

Next, we conducted multivariate analysis to further identify the prominently independent prognostic factors for OS. The significant indicators in
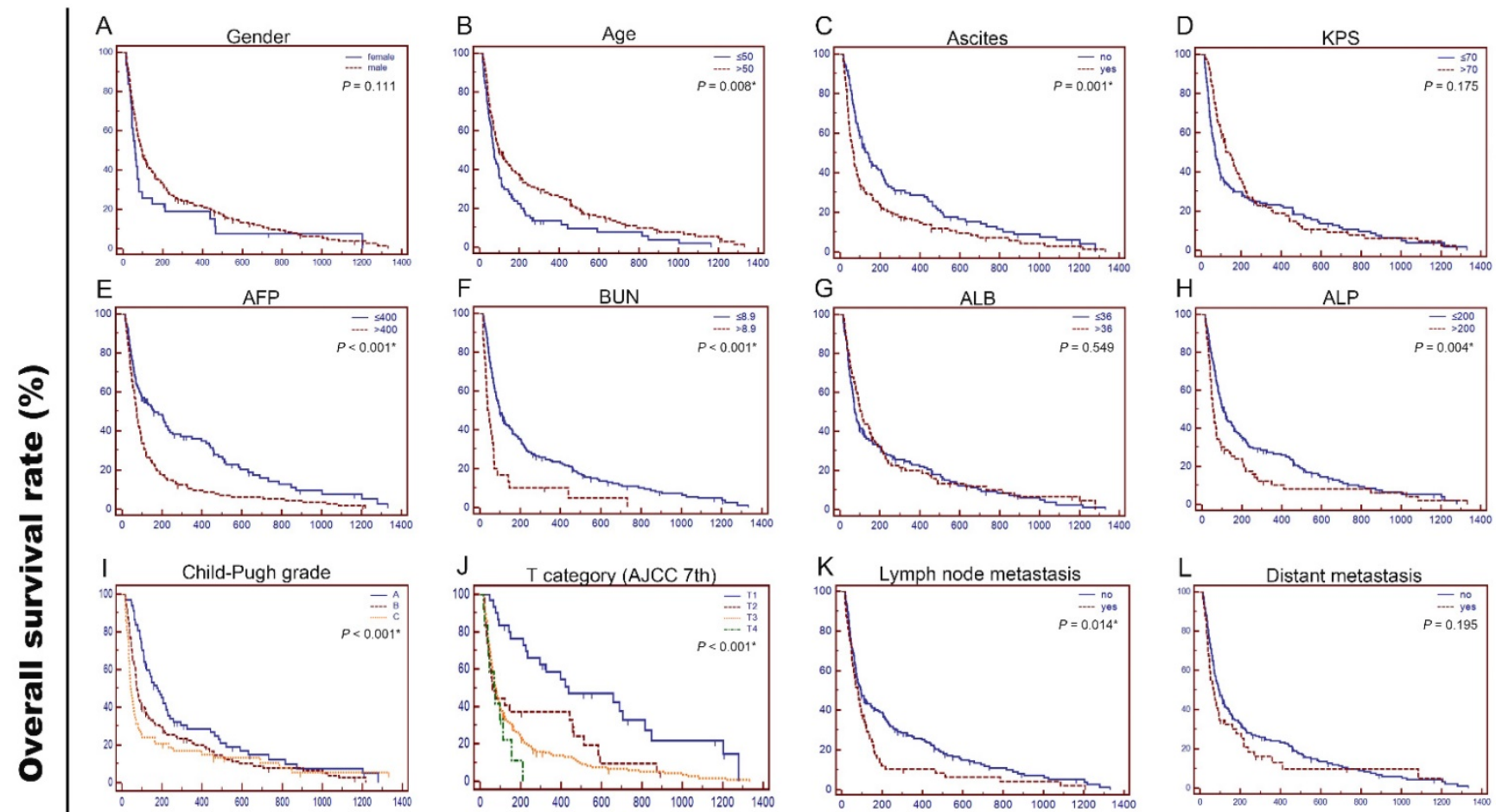
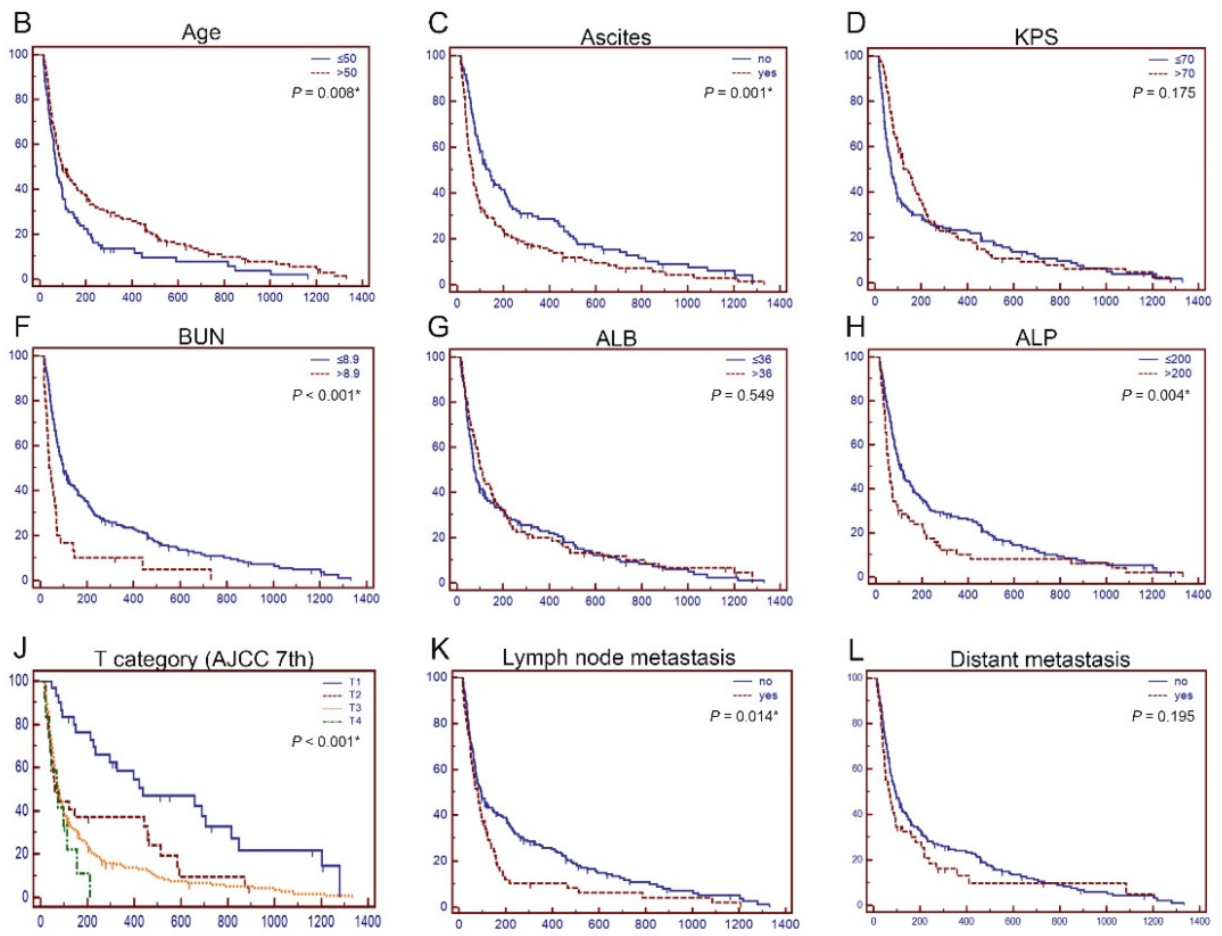

the univariate analyses were then included in Cox proportional hazards model using a backward stepwise method. As shown in Table 3, AAPR was indicated as an independent prognostic index $(H R=$ $0.592, P=0.007)$, implying that AAPR larger than 0.38 was correlated with a survival benefit or longer survival time. Other independent factors in this study contained age, serum AFP and BUN levels, Child-Pugh grade and T category.

Previous studies have reported that pretreatment level of ALB and ALP were both prognostic factors for HCC [23, 24]. Comparison of ROC curves revealed that area under curve (AUC) of AAPR was relatively larger than that of ALB or ALP alone in predicting OS time ( 0.619 vs 0.535 vs 0.543 for AAPR, ALB and ALP, respectively), which implied that AAPR served better than ALB or ALP alone as a prognostic indicator for OS in this study (Figure 3).

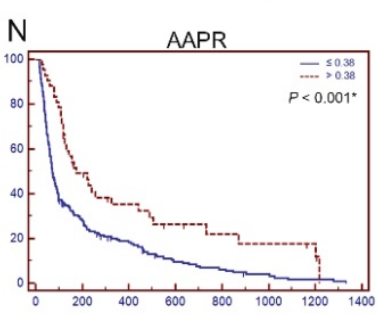

\section{Time after diagnosis (days)}

Figure 2. Kaplan-Meier curves for overall survival (OS) were stratified by different features of 237 patients with advanced $\mathrm{HCC}$ in present study. Clinicopathological features included gender (A), age (B), ascites (C), KPS (D), AFP, ng/dL (E), BUN, mmol/L (F), ALB, g/L (G), ALP, U/L (H), Child-Pugh grade (I), T category (AJCC 7th) (J), lymph node metastasis $(K)$, distant metastasis $(L)$, PVTT $(M)$, and AAPR $(N)$, respectively. OS was significantly different among subgroups stratified by all values (all $P<0.05)$ except gender $(P=0.111)$, KPS $(P=0.175)$, ALB $(P=0.549)$, and distant metastasis $(P=0.195)$. KPS: Karnofsky performance status; AFP: alpha fetoprotein; BUN: blood urea nitrogen; ALB: albumin; ALP: alkaline phosphatase; AJCC: American Joint Committee on Cancer; AAPR: albumin-to-alkaline phosphatase ratio; PVTT: portal vein tumor thrombus. 
Table 3. Univariate and multivariate analyses of prognostic factors for overall survival of patients with advanced HCC in present study.

\begin{tabular}{|c|c|c|c|c|c|c|c|}
\hline \multirow[t]{2}{*}{ Variable } & \multicolumn{2}{|l|}{ Univariate } & \multicolumn{5}{|c|}{ Multivariate } \\
\hline & Log-rank $\chi^{2}$ & $P$ value & $B$ & $S E$ & HR & $95 \% \mathrm{CI}$ & $P$ value \\
\hline Gender (male/female) & 2.540 & 0.111 & & & & & \\
\hline Age, years $(>50 / \leq 50)$ & 7.021 & $0.008^{*}$ & -0.367 & 0.150 & 0.693 & 0.517 to 0.929 & 0.015 \\
\hline Ascites (Yes/no) & 10.709 & $0.001^{*}$ & & & & & \\
\hline $\operatorname{KPS}(\geq 70 /<70)$ & 1.844 & 0.175 & & & & & \\
\hline $\mathrm{AFP}, \mathrm{ng} / \mathrm{dL}(>400 / \leq 400)$ & 18.330 & $<0.001^{*}$ & 0.584 & 0.151 & 1.794 & 1.336 to 2.409 & $<0.001$ \\
\hline 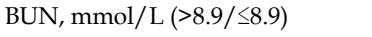 & 19.479 & $<0.001^{*}$ & 0.988 & 0.210 & 2.685 & 1.782 to 4.045 & $<0.001$ \\
\hline ALB, g/L $(>36 / \leq 36)$ & 0.360 & 0.549 & & & & & \\
\hline ALP, U/L $(>200 / \leq 200)$ & 8.415 & $0.004^{*}$ & & & & & \\
\hline Child-Pugh grade & 12.878 & $<0.001^{*}$ & & & & & 0.006 \\
\hline A & & & NA & NA & 1 & Reference & NA \\
\hline B & & & 0.156 & 0.178 & 1.168 & 0.826 to 1.653 & 0.382 \\
\hline $\mathrm{C}$ & & & 0.477 & 0.174 & 1.611 & 1.148 to 2.263 & 0.006 \\
\hline T category (AJCC 7th) & 24.259 & $<0.001^{*}$ & & & & & $<0.001$ \\
\hline $\mathrm{T} 1$ & & & NA & NA & 1 & Reference & NA \\
\hline $\mathrm{T} 2$ & & & 0.934 & 0.301 & 2.545 & 1.416 to 4.573 & 0.002 \\
\hline T3 & & & 1.053 & 0.236 & 2.867 & 1.810 to 4.542 & $<0.001$ \\
\hline $\mathrm{T} 4$ & & & 1.302 & 0.385 & 3.676 & 1.736 to 7.783 & $<0.001$ \\
\hline Lymph node metastasis (yes/no) & 6.100 & $0.014^{*}$ & & & & & \\
\hline Distant metastasis (yes/no) & 1.676 & 0.195 & & & & & \\
\hline PVTT (yes/no) & 28.866 & $<0.001^{*}$ & & & & & \\
\hline $\operatorname{AAPR}(>0.38 / \leq 0.38)$ & 13.222 & $<0.001^{*}$ & -0.524 & 0.195 & 0.592 & 0.405 to 0.866 & 0.007 \\
\hline
\end{tabular}

B: beta value; SE: standard error; HR: hazard ratio; CI: confidence interval; NA: not applicable; KPS: Karnofsky performance status; AFP: alpha fetoprotein; BUN: blood urea nitrogen; ALB: albumin; ALP: alkaline phosphatase; AAPR: albumin-to-alkaline phosphatase ratio; AJCC: American Joint Committee on Cancer; PVTT: portal vein tumor thrombus.

*Variables with $P$ value less than 0.05 were entered into multivariate analyses.

\section{Comparison of AAPR-TNM and TNM staging systems}

For more reliable outcomes, we integrated AAPR with TNM staging system. OS curves stratified by TNM and AAPR-TNM systems were showed in Figure 4-A\&B. The median survival time of patients in AAPR-TNM grade 4 to 5 was shorter than 3 months. Comparison of ROC curves exhibited that the AUC of AAPR-TNM system was significantly larger than that of TNM system when predicting 3-month survival time $(0.670$ vs $0.611, P<0.01)$ (Figure $4-C)$. As for overall survival, LRT indicated that AAPR-TNM system has a significantly larger $\chi^{2}(26.4$ vs $16.4, P<$ 0.01 ), and a significantly smaller Akaike information criterion (AIC) value (1936 vs 1948, $P<0.01)$ comparing with TNM system, which indicated that AAPR-TNM system outperformed TNM system in predicting OS time (Table 4).

\section{Discussion}

Our present study mainly concentrated on evaluating the prognostic value of AAPR in advanced HCC patients without receiving any standard anti-cancer therapies. These patients reflected a natural progress of HCC and were worth being studied. Our investigation implied that AAPR was an independent prognostic index in advanced HCC; AAPR-TNM system precedes TNM system in predicting overall survival.

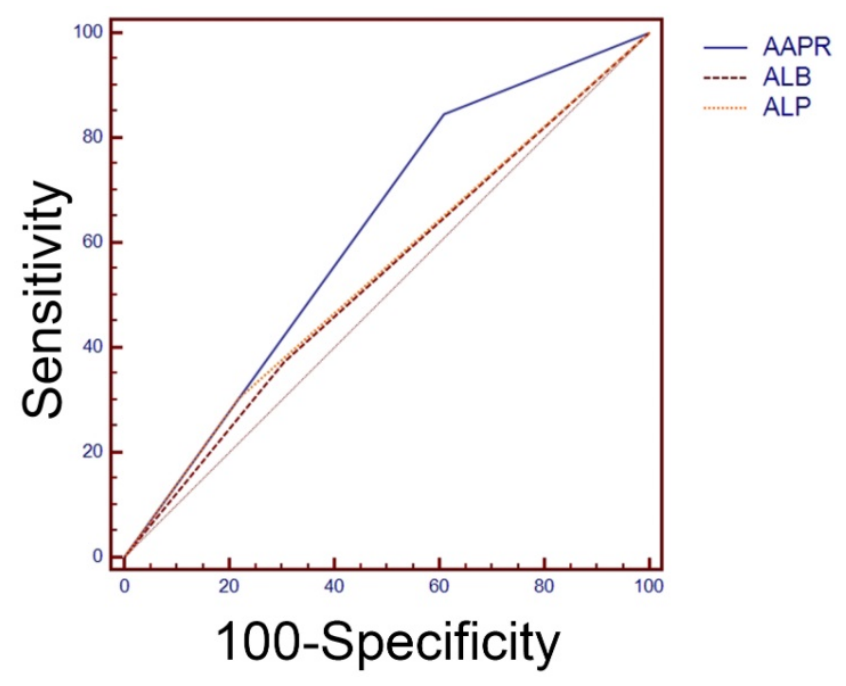

Figure 3. Comparisons of the area under curve (AUC) for survival status among AAPR, ALB and ALP using receiver operating characteristic curves. The AUC of AAPR, ALB and ALP were $0.619,0.535$ and 0.543 , respectively. AAPR: albumin-to-alkaline phosphatase ratio; ALB: albumin; ALP: alkaline phosphatase.

Table 4. Comparisons of the value of TNM and AAPR-TNM systems in predicting prognosis of overall survival among the patients in present study.

\begin{tabular}{llcc}
\hline Staging system & Likelihood ratio test $\chi^{2}$ & AIC & $P$ value \\
\hline TNM & 16.4 & 1948 & $<0.01$ \\
AAPR-TNM & 26.4 & 1936 & $<0.01$ \\
\hline AAPR: albumin-to-alkaline phosphatase ratio; TNM: tumor-node-metastasis; AIC:
\end{tabular}

Akaike information criterion. 


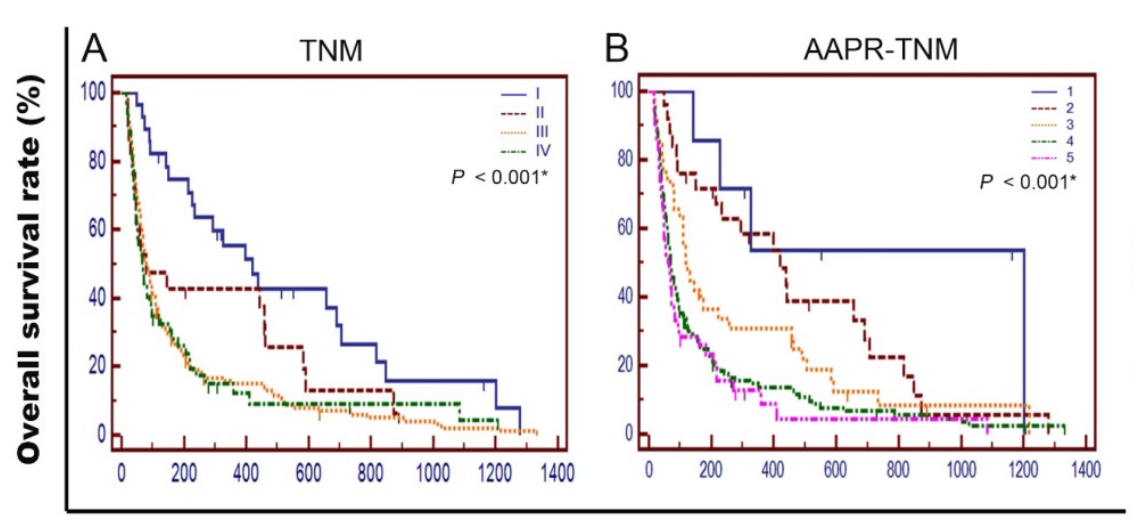

Time after diagnosis (days)

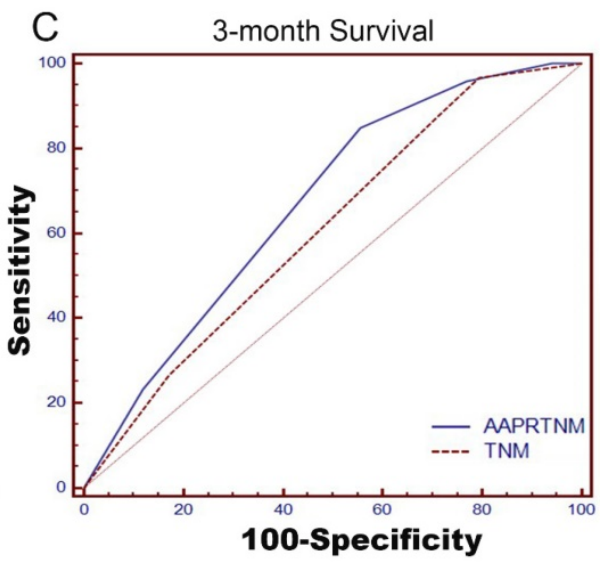

Figure 4. Comparisons of AAPR-TNM and TNM staging systems in prognosis prediction of 237 advanced HCC patients in present study. Survival curves were stratified by TNM (A), and AAPR-TNM (B), respectively. OS was significantly different among subgroups stratified by the above variables (both $P<0.001)$. Comparisons of the area under curve (AUC) for 3-month survival prediction between AAPR-TNM and TNM systems using ROC curve (C). The AUC of AAPR-TNM and TNM systems were 0.670 and 0.611 , respectively. TNM: Tumor-node-metastasis; AAPR: albumin-to-alkaline phosphatase ratio.

Liver function test is an economically effective and easily accessible laboratory test for assessing liver function in clinical practice. Two important items of this test are serum levels of ALB and ALP. Serum ALB level mainly reflects the protein synthetic capability of liver, and is influenced by both fundamental chronic liver dysfunction and systemic immunological response to either tumor itself or inflammatory microenvironment in HCC [25]. As an essential index of nutrition, ALB was reported with the ability to stabilize cell growth and proliferation [26], exert antioxidant reaction to against carcinogenesis [27], and modulate immune reaction to ameliorate liver function [28]. Therefore, low level of ALB not only indicates insufficient hepatic function, but also reflects underperformance of human defense including cellular and humoral immunity, which possibly results in cancer patients' inert response to anti-cancer therapies. ALP is a hydrolytic enzyme that dephosphorylates and transphosphorylates molecules including nucleotides (adenosine triphosphate, adenosine biphosphate), pathogen-associated molecule patterns and danger-associated molecule patterns [29]. With multiple isoforms, ALP exists throughout the human body, but mainly in the liver, bile duct, bone and placenta. The serum ALP level increases during pregnancy or under some pathological conditions including cholangiocarcinoma, HCC, biliary cirrhosis and liver injury [30]. The level of ALP was reported as an independent prognostic biomarker for patients with HCC [23] and as a variable integrated into some clinical staging systems such as the Chinese University Prognostic Index (CUPI) system to predict survival [31].

First reported by Anthony and colleagues, AAPR performed as an independent predictor of progress-free survival and overall survival for HCC patients who underwent curative surgery or palliative anti-cancer therapies [19]. Nevertheless, the prognostic role of AAPR in advanced HCC patients without receiving any standard anti-cancer treatments, remains unknown. In our present study, the level of AAPR was significantly lower in patients with ascites, or with PVTT as opposed to those without. Similar results were exhibited in patients being Child-Pugh grade $\mathrm{B} \& \mathrm{C}$, or with KPS $<70$ comparing with those being Child-Pugh grade $\mathrm{A}$, or with $\mathrm{KPS} \geq 70$. A 0.38 cutoff value of AAPR was applied in predicting survival in this study. AAPR less than 0.38 was correlated with higher frequencies of ascites, PVTT, Child-Pugh grade B\&C, and KPS < 70 , and more importantly, shorter median survival time. Both univariate and multivariate analyses indicated an independent prognostic role of AAPR in predicting OS. Comparison of ROC curves implied that AAPR preceded ALB or ALP alone as a prognostic index for OS in advanced HCC. Furthermore, we integrated AAPR with TNM system and found that AAPR-TNM system outperformed TNM system in predicting OS, which might help clinical decision-making in regards of executing targeted treatments and immunotherapy. The median survival of patients in AAPR-TNM grade 4 to 5 was shorter than 3 months, which means these patients might not be suitable for clinical trials. However, independent cohorts of advanced HCC are needed to further confirm the practical utility of AAPR-TNM staging system.

To our knowledge, this is the first study focusing on validating the prognostic potential of AAPR in advanced HCC without receiving standard anti-cancer therapy. AAPR is a simple but discriminatory and objective variable, and is easier 
employed in clinical practice. Moreover, association of AAPR and other clinicopathological characteristics were explored in this study. Last but not least, all enrolled patients did not receive any standard anti-cancer treatments, which eliminated interruptions from anti-cancer treatment modalities and exhibited a relatively natural development of HCC.

However, some limitations of this study must be clarified. Firstly, as a retrospective study, selection bias still exists though both inclusion and exclusion criteria were strictly performed. Secondly, the number of patients included in this study was small, and all patients were from one single center. Thirdly, a lack of validation cohorts limits the further confirmation of AAPR as an independent indicator for patients with advanced HCC. As an index combined by ALB and ALP, low AAPR may reflect a poor nutrition status, exhausted liver function, inactive immune reaction, and invasive tumor form, all of which may lead to poor prognosis. But molecular mechanisms of the correlation between AAPR and prognosis need to be further elucidated.

To sum up, our present study suggested that AAPR was a potentially valuable prognostic index in advanced HCC patients without receiving standard anti-cancer therapy. AAPR-TNM system precedes TNM system in predicting overall survival in present study. Larger scale and multi-center research is warranted for further confirmation.

\section{Abbreviations}

HCC: hepatocellular carcinoma; KPS: Karnofsky performance status; AFP: alpha fetoprotein; BUN: blood urea nitrogen; ALB: albumin; ALP: alkaline phosphatase; AAPR: albumin-to-alkaline phosphatase ratio; TNM: Tumor-node-metastasis; AJCC: American Joint Committee on Cancer; OS: overall survival; ROC curve: receiver operating characteristics curve.

\section{Acknowledgements}

This study was supported by grants from the National Natural Science Foundation of China (grant no. 31600710 and 81372374), the Combination Project of Production, the scientific research project for University belong to Guangzhou Municipal (grant no. 1201630019), and Natural Science Foundation of Guangdong (grant no. 2014A030313146 and 2016A030313302) and Project on the Integration of Industry, Education and Research of Guangdong Province (grant no. 2012B091100460).

\section{Competing Interests}

The authors of this study have no disclosures regarding funding or any conflict of interest with respect to this manuscript. The authors have not received fees for serving as speakers for any organization, and have not received research funding from any organization. The authors are not employees of any organization and do not own stocks and/or shares in any organization. The authors do not own patents.

\section{References}

1. Torre LA, Bray F, Siegel RL, Ferlay J, Lortet-Tieulent J, Jemal A. Global cancer statistics, 2012. CA: a cancer journal for clinicians. 2015; 65: 87-108.

2. European Association For The Study Of The Liver, European Organisation For Research, Treatment Of Cancer. EASL-EORTC clinical practice guidelines: management of hepatocellular carcinoma. Journal of hepatology. 2012; 56: 908-43.

3. Chen W, Zheng R, Baade PD, Zhang S, Zeng H, Bray F, et al. Cancer statistics in China, 2015. CA: a cancer journal for clinicians. 2016; 66: 115-32.

4. Bruix J, Qin S, Merle P, Granito A, Huang YH, Bodoky G, et al. Regorafenib for patients with hepatocellular carcinoma who progressed on sorafenib treatment (RESORCE): a randomised, double-blind, placebo-controlled, phase 3 trial. Lancet. 2017; 389: 56-66.

5. Cheng BQ, Jia CQ, Liu CT, Fan W, Wang QL, Zhang ZL, et al. Chemoembolization combined with radiofrequency ablation for patients with hepatocellular carcinoma larger than $3 \mathrm{~cm}$ : a randomized controlled trial. Jama. 2008; 299: 1669-77.

6. Granito A, Bolondi L. Non-transplant therapies for patients with hepatocellular carcinoma and Child-Pugh-Turcotte class B cirrhosis. The Lancet Oncology. 2017; 18: e101-e12.

7. Omata M, Cheng AL, Kokudo N, Kudo M, Lee JM, Jia J, et al. Asia-Pacific clinical practice guidelines on the management of hepatocellular carcinoma: a 2017 update. Hepatol Int. 2017; 11: 317-370.

8. Qasim W, Brunetto M, Gehring AJ, Xue SA, Schurich A, Khakpoor A, et al. Immunotherapy of HCC metastases with autologous T cell receptor redirected $\mathrm{T}$ cells, targeting HBsAg in a liver transplant patient. Journal of hepatology. 2015; 62: 486-91.

9. Zhou SL, Zhou ZJ, Hu ZQ, Huang XW, Wang Z, Chen EB, et al. Tumor-Associated Neutrophils Recruit Macrophages and T-Regulatory Cells to Promote Progression of Hepatocellular Carcinoma and Resistance to Sorafenib. Gastroenterology. 2016; 150: 1646-58 e17.

10. Zhou L, Rui JA, Wang SB, Chen SG, Qu Q. Risk factors of microvascular invasion, portal vein tumor thrombosis and poor post-resectional survival in HBV-related hepatocellular carcinoma. Hepato-gastroenterology. 2014; 61: 1696-703.

11. Hiraoka A, Kumada T, Kudo M, Hirooka M, Tsuji K, Itobayashi E, et al. Albumin-Bilirubin (ALBI) Grade as Part of the Evidence-Based Clinical Practice Guideline for HCC of the Japan Society of Hepatology: A Comparison with the Liver Damage and Child-Pugh Classifications. Liver Cancer. 2017; 6: 204-15.

12. Notarpaolo A, Layese R, Magistri P, Gambato M, Colledan M, Magini G, et al. Validation of the AFP model as a predictor of HCC recurrence in patients with viral hepatitis-related cirrhosis who had received a liver transplant for HCC. Journal of hepatology. 2017; 66: 552-9.

13. Zhu AX, Chen D, He W, Kanai M, Voi M, Chen LT, et al. Integrative biomarker analyses indicate etiological variations in hepatocellular carcinoma. Journal of hepatology. 2016; 65: 296-304

14. Carr BI, Guerra V. Hepatocellular Carcinoma Extrahepatic Metastasis in Relation to Tumor Size and Alkaline Phosphatase Levels. Oncology. 2016; 90: 136-42.

15. Llovet JM, Bru C, Bruix J. Prognosis of hepatocellular carcinoma: the BCLC staging classification. Seminars in liver disease. 1999; 19: 329-38.

16. Reig M, Darnell A, Forner A, Rimola J, Ayuso C, Bruix J. Systemic therapy for hepatocellular carcinoma: the issue of treatment stage migration and registration of progression using the BCLC-refined RECIST. Seminars in liver disease. 2014; 34: 444-55.

17. Kee KM, Wang JH, Lin CY, Wang CC, Cheng YF, Lu SN. Validation of the 7th edition TNM staging system for hepatocellular carcinoma: an analysis of 8,828 patients in a single medical center. Digestive diseases and sciences. 2013; 58: 2721-8.

18. Nie M, Sun P, Chen C, Bi X, Wang Y, Yang H, et al. Albumin-to-Alkaline Phosphatase Ratio: A Novel Prognostic Index of Overall Survival in Cisplatin-based Chemotherapy-treated Patients with Metastatic Nasopharyngeal Carcinoma. J Cancer. 2017; 8: 809-15.

19. Chan AW, Chan SL, Mo FK, Wong GL, Wong VW, Cheung YS, et al. Albumin-to-alkaline phosphatase ratio: a novel prognostic index for hepatocellular carcinoma. Disease markers. 2015; 2015: 564057.

20. Bruix J, Sherman M, Practice Guidelines Committee, American Association for the Study of Liver Diseases. Management of hepatocellular carcinoma. Hepatology (Baltimore, Md). 2005; 42: 1208-36.

21. NCCN. National Comprehensive Cancer Network (NCCN) Clinical Practice Guidelines in Oncology. Hepatobiliary Cancers, Version 2; 2015. 
22. Fluss R, Faraggi D, Reiser B. Estimation of the Youden Index and its associated cutoff point. Biometrical journal Biometrische Zeitschrift. 2005; 47: 458-72.

23. Yu MC, Chan KM, Lee CF, Lee YS, Eldeen FZ, Chou HS, et al. Alkaline phosphatase: does it have a role in predicting hepatocellular carcinoma recurrence? Journal of gastrointestinal surgery : official journal of the Society for Surgery of the Alimentary Tract. 2011; 15: 1440-9.

24. Cho HJ, Kim B, Lee JD, Kang DR, Kim JK, Lee JH, et al Development of Risk Prediction Model for Hepatocellular Carcinoma Progression of Indeterminate Nodules in Hepatitis B Virus-Related Cirrhotic Liver. The American journal of gastroenterology. 2017; 112: 460-70.

25. Tanriverdi O. A discussion of serum albumin level in advanced-stage hepatocellular carcinoma: a medical oncologist's perspective. Med Oncol. 2014; 31: 282.

26. Nojiri S, Joh T. Albumin suppresses human hepatocellular carcinoma proliferation and the cell cycle. International Journal of Molecular Sciences. 2014; 15: 5163-74.

27. Reebye V, Saetrom P, Mintz PJ, Huang KW, Swiderski P, Peng L, et al. Novel RNA oligonucleotide improves liver function and inhibits liver carcinogenesis in vivo. Hepatology (Baltimore, Md). 2014; 59: 216-27.

28. Garcia-Martinez R, Andreola F, Mehta G, Poulton K, Oria M, Jover M, et al. Immunomodulatory and antioxidant function of albumin stabilises the endothelium and improves survival in a rodent model of chronic liver failure. Journal of hepatology. 2015; 62: 799-806.

29. Lalles JP. Intestinal alkaline phosphatase: multiple biological roles in maintenance of intestinal homeostasis and modulation by diet. Nutrition reviews. 2010; 68: 323-32

30. Williamson KD, Chapman RW. Editorial: further evidence for the role of serum alkaline phosphatase as a useful surrogate marker of prognosis in PSC. Alimentary pharmacology \& therapeutics. 2015; 41: 149-51.

31. Leung TW, Tang AM, Zee B, Lau WY, Lai PB, Leung KL, et al. Construction of the Chinese University Prognostic Index for hepatocellular carcinoma and comparison with the TNM staging system, the Okuda staging system, and the Cancer of the Liver Italian Program staging system: a study based on 926 patients. Cancer. 2002; 94: 1760-9. 